Published ahead of print on March 4, 2022, as doi:10.5045/br.2021.2021164

Copyright (c) 2022 Korean Society of Hematology

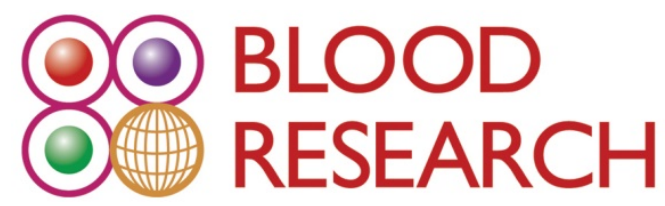

Clinical characteristics and treatment outcomes of children and adolescents with aggressive mature B-cell lymphoma: a single-center analysis

Woojung Jeon, Young Kwon Koh, Sunghan Kang, Hyery Kim, Kyung-Nam Koh, Ho Joon Im

Citation: Jeon $W$, Koh $Y K$, Kang $S$, Kim H, Koh KN, Im HJ. Clinical characteristics and treatmen t outcomes of children and adolescents with aggressive mature B-cell lymphoma: a single-cent er analysis. Blood Res 2022. [Epub ahead of print]

Publisher's disclaimer: This manuscript has completed peer review and has been accepted for publication. We are distributing the authors' version of manuscript prior to technical and Engli sh editing, typesetting and proof reading. A final version approved by the authors' will follow in a regular issue of BLOOD RESEARCH. All legal disclaimers that apply to BLOOD RESEARCH also pertain to this production process. 


\section{Clinical characteristics and treatment outcomes of children and adolescents with aggressive mature B-cell lymphoma: a single-center analysis}

Woojung Jeon ${ }^{1}$, Young Kwon Koh ${ }^{1,2}$, Sunghan Kang ${ }^{1,2}$, Hyery Kim ${ }^{1,2}$, Kyung-Nam Koh ${ }^{1,2}$, Ho Joon $\operatorname{Im}^{1,2}$

${ }^{1}$ Department of Pediatrics, University of Ulsan College of Medicine, Asan Medical Center, ${ }^{2}$ Division of Pediatric Hematology/Oncology, Department of Pediatrics, University of Ulsan College of Medicine, Asan Medical Center, Seoul, Korea

Correspondence to: Hyery Kim, M.D., Ph.D.

Division of Pediatric Hematology/Oncology, Department of Pediatrics, University of Ulsan College of Medicine, Asan Medical Center, 88-1 Olympic-ro 43-gil, Songpa-gu, Seoul 05505, Korea

E-mail: taban@hanmail.net

This study was supported by a National Research Foundation of Korea grant, funded by the Korean government (Ministry of Science and ICT) (no. NRF-2018R1C1B5047092). 


\begin{abstract}
Background: Aggressive mature B-cell non-Hodgkin lymphoma (B-NHL) is the most common nonHodgkin lymphoma in children. The outcome of chemotherapy for B-NHL has improved over decades.

Methods: We reviewed 82 children and adolescents with B-NHL diagnosed at Asan Medical Center between 1993 and 2020. The D-COMP/COMP (daunomycin-cyclophosphamide, doxorubicin, vincristine, and prednisolone), Pediatric Oncology Group (POG)-9219/9315/9317, R-CHOP/CHOP (rituximab-cyclophosphamide, doxorubicin, vincristine, and prednisolone), and Lymphomes Malins B 89 (LMB89)/LMB96 regimens were administered. In 2018, rituximab was added to the LMB protocol (R-LMB) for advanced-staged Burkitt lymphoma (BL). The patients' clinical features and treatment outcomes were retrospectively analyzed.
\end{abstract}

Results: The most common subtype was BL (61\%), followed by diffuse large B-cell lymphoma (DLBCL) (35\%). The median age was 7.8 (range, 1.3-16.4) years, and the most frequently used regimen was French-American-British (FAB)/LMB96 (58 patients, 70.7\%). The 5-year overall survival (OS) and event-free survival (EFS) rates were $92.5 \%$ and $85.7 \%$, respectively. The EFS rates of patients with BL and DLBCL were $90.0 \%$ and $79.3 \%$, respectively. Among the FAB/LMB risk groups, group C $(85.7 \%)$ had a significantly lower 5-year OS $(P=0.037)$. Eleven events occurred (6 relapses, 3 deaths, and 2 secondary malignancies) during the median follow-up of 7.1 (range, 3.7118.5) months. Two patients treated with R-LMB had good outcomes without complications.

Conclusion: Various treatment regimens have favorable outcomes in pediatric patients with B-NHL. However, further studies are needed to improve survival in high-risk patients. In addition, careful monitoring for acute toxicity or secondary malignancy due to intensive multidrug chemotherapy is required.

Keywords: Mature B-cell lymphoma, Children, Survival, LMB protocol, Rituximab 


\section{INTRODUCTION}

Non-Hodgkin lymphoma (NHL) is a diverse group of lymphoid neoplasms categorized according to the characteristics of lymphoma cells (B, T, and natural killer cells), cell morphology, immunophenotype, genetic features, and prognosis. Children generally present with high-grade lymphomas that tend to be aggressive and fast growing, such as Burkitt lymphoma (BL), diffuse large B-cell lymphoma (DLBCL), lymphoblastic lymphoma, and anaplastic large cell lymphoma. Among them, aggressive mature B-cell lymphomas account for approximately $60 \%$ of NHLs in children [1]. They include many subtypes and variants, such as DLBCL, BL, and high-grade B-cell lymphoma, not otherwise specified (HGBL-NOS) [2].

Over the recent decades, the outcome of treatment with short-duration, dose-intensive, and stagebased multiagent chemotherapy in pediatric patients with mature B-cell lymphomas has considerably improved, with survival rates of $>80 \%[3,4]$. Since the 1980s, 3 major US and European childhood cancer groups have contributed to the most effective and proven treatment approaches to date: Lymphomes Malins B (LMB) group, Berlin-Frankfurt-Münster (BFM) group, and Children's Cancer Group/Children's Oncology Group (CCG/COG). The French-American-British (FAB)/LMB studies divided patients into 3 groups based on disease stage and resection status. The FAB/LMB protocols are based on the COPAD regimen (cyclophosphamide, vincristine, prednisolone, and doxorubicin). Central nervous system (CNS) prophylaxis based on high-dose (HD) methotrexate (MTX) and intrathecal injections of MTX has been proven effective $[3,5,6]$.

The BFM protocols are similar to the $\mathrm{FAB} / \mathrm{LMB}$ protocols, with differences in the details of risk group stratification and treatment intensity. In BFM studies, patients were stratified into 4 risk groups according to stage, resection of localized tumors, lactate dehydrogenase (LDH) level, and CNS involvement [7]. BFM studies revealed that patients with advanced disease benefit from more prolonged HD MTX exposure and HD cytarabine. As the LMB and BFM protocols have provided dramatic improvements in treatment outcomes in pediatric NHL, they have been the most often used treatment strategies. Recently, rituximab was added to the standard LMB chemotherapy (R-LMB), and 
this combination has shown efficacy in children and adolescents with high-grade, high-risk, mature Bcell NHL (B-NHL) [8].

Since the introduction of effective intensive multiagent chemotherapy, very few children and adolescents (5-15\%) with mature B-cell lymphoma have experienced refractory or relapsing disease. However, the prognosis of these patients remains poor, with survival rates of $<30 \%$ [9]. Several studies have aimed to identify risk factors to determine the prognosis of these patients. Increased serum LDH levels and CNS involvement are considered factors associated with a poor prognosis [10]. For these groups of patients, multiple strategies have been proposed and new therapies are being investigated [1].

The aim of our study was to investigate the clinical characteristics and treatment outcomes of children and adolescents with mature B-cell lymphoma at a single center.

\section{MATERIALS AND METHODS}

\section{Patients}

We retrospectively reviewed the clinical records of 82 pediatric patients with aggressive mature Bcell lymphomas (BL, DLBCL, or HGBL-NOS) who were diagnosed and treated at Asan Medical Center Children's Hospital between March 1993 and March 2020.

The diagnosis was made on the basis of tissue biopsy and cerebrospinal fluid (CSF) or effusion analysis and classified according to the World Health Organization (WHO) classification of lymphoid neoplasms [2]. Disease staging was performed according to the St. Jude staging system [11], which includes physical examination, peripheral blood smear examination, bone marrow (BM) biopsy, CSF analysis, serum LDH level measurement, computed tomography (CT) or magnetic resonance imaging, and positron emission tomography (PET). BM involvement was defined as the presence of tumor cells on a BM smear. CNS involvement was confirmed in the presence of at least one of the following: any lymphoma cells identified with CSF cytology, intracranial or parameningeal lesions on imaging evaluations, cranial nerve palsy, or clinical spinal cord compression. We also assessed the FAB/LMB risk groups according to protocol-defined classifications [12]. This study was approved by the 
Institutional Review Board of Asan Medical Center (approval no. S2021-2179).

\section{Statistical analysis}

The rates of overall survival (OS) and event-free survival (EFS) were estimated using the KaplanMeier method and compared using the log-rank test. OS was defined as the time from the date of diagnosis to the date of death of any cause or the date of the last follow-up visit. EFS was defined as the time from the date of diagnosis to the date of the first relapse, progression, secondary malignancy, death, or the last follow-up visit. All analyses were performed using SPSS (version 27.0, IBM Corp., Armonk, NY, USA), and the threshold for statistical significance was set at $P<0.05$.

\section{RESULTS}

\section{Patient characteristics}

The demographic and clinical characteristics of the 82 patients are summarized in Table 1 . The median age was 7.8 years (range, $1.3-16.4 \mathrm{yr}$ ), with a male predominance (males vs. females, $79 \%$ vs. $21 \%)$. BL (61.0\%) was the most common pathologic subtype, followed by DLBCL (35.4\%) and HGBL-NOS (3.6\%). According to the St. Jude classification, 4 (4.9\%), 26 (31.7\%), 30 (36.6\%), and $22(26.8 \%)$ patients had stage I, II, III, and IV disease, respectively. At diagnosis, BM involvement was present in 15 patients and CNS involvement was observed in 8 patients. Forty-three patients (52.4\%) had primary tumors in the abdomen, whereas 17 patients $(20.7 \%)$ had primary tumors in the head and neck. Only 4 patients had primary masses in the conjunctiva or bone.

\section{Treatment}

The 82 patients were treated using a variety of chemotherapy protocols (Table 1, Fig. 1). From the 1990s to the early 2000s, the initial treatment was as follows: 3 patients received the D-COMP/COMP (daunomycin-cyclophosphamide, doxorubicin, vincristine, and prednisolone) regimens [13]; 3 patients received the Pediatric Oncology Group (POG)-9219 regimen [14]; 3 patients received the POG-9315 regimen [15]; and 3 patients received the POG-9317 regimen [16]. Two patients with 
DLBCL received the R-CHOP/CHOP (rituximab-cyclophosphamide, doxorubicin, vincristine, and prednisolone) regimens. After 1998, the LMB backbone protocols (LMB89, FAB/LMB96) [5, 6] have mainly been used. Nine patients received the LMB89 regimen, and 58 patients received the FAB/LMB96 regimen. In 2018, we started using the R-LMB protocol in patients with BL classified as group C [8], and 2 such patients were included in this study.

\section{Survival}

As of February 2021, the 5-year OS and EFS rates were $92.5 \pm 2.9 \%$ and $85.7 \pm 4.1 \%$, respectively, with a median follow-up duration of 136.4 months (range, 3.7-344.2 mo; Fig. 2). According to the histopathologic subtypes, the 5-year OS rate was $100 \%$ for patients with HGBL, $91.9 \pm 3.9 \%$ for patients with BL, and $92.6 \pm 5.0 \%$ for patients with DLBCL. The difference in the 5-year OS among the 3 subtypes was not statistically significant $(P=0.882)$ (Fig. 3). The 5 -year EFS rate was $100 \%$ for patients with $\mathrm{HGBL}, 89.9 \pm 4.3 \%$ for patients with $\mathrm{BL}$, and $82.2 \pm 7.2 \%$ for patients with DLBCL ( $P=0.336)$. When comparing survival rates according to the initial stage, a tendency for lower OS and EFS was observed in those with advanced disease stages, especially in patients with DLBCL. The OS and EFS rates of patients with stage IV DLBCL were only $66.7 \%$ and $33.3 \%$, respectively (Fig. 4). However, no statistically significant difference was found in the overall trends [5-year OS of patients with BL $(P=0.929)$ and DLBCL $(P=0.199)$; 5-year EFS of patients with BL $(P=0.726)$ and DLBCL $(P=0.198)]$. We also investigated the survival rates according to risk groups in patients with $\mathrm{BL}$ and DLBCL treated with the LMB protocols (Fig. 5). The 5-year OS of patients in the group C risk group

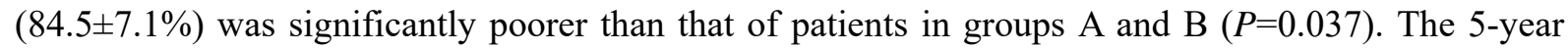
EFS was also low in patients in group C (76.5 $\pm 8.4 \%)$; however, the differences were not statistically significant $(P=0.081)$.

According to the initial protocol, no statistically significant difference was observed in both OS $(P=0.179)$ and EFS $(P=0.332)$. The 5-year OS rate was $100 \%, 94.7 \pm 3.0 \%, 88.9 \pm 10.5 \%, 88.9 \pm 10.5 \%$, and $50.0 \pm 35.4 \%$ and the EFS rate was $100 \%, 89.5 \pm 4.1 \%, 88.9 \pm 10.5 \%, 77.8 \pm 13.9 \%$, and $50.0 \pm 35.4 \%$ in patients treated with the D-COMP/COMP, LMB96, LMB89, POG, and R-CHOP/CHOP regimens, 
respectively (Fig. 6).

\section{Events}

A total of 11 events occurred during the follow-up period (Table 2).

1) Relapse

Six patients (7.3\%) experienced disease relapse at a median of 6.8 months (range, 4.3-13.9 mo) from the initial diagnosis. Of these 6 patients, 2 had stage II disease, 3 had stage III disease, and 1 had stage IV disease at diagnosis. All patients with recurrence were treated with salvage chemotherapy. Three patients with local relapse survived after salvage chemotherapy with CCG-5941, COGANHL0121, or POG-9317 (Fig. 1).

Three of the patients who experienced relapse eventually died. A 4-year-old female patient with BL had peritoneal and bone masses with BM involvement. She had CNS relapse after the completion of the initial LMB96 group C regimen. St. Jude Children's Research Hospital Total Therapy Protocol XV and additional radiotherapy ( 20 Gy delivered in 10 fractions to the whole body) were administered as rescue treatment; however, the BM lymphoma relapsed [17]. Eventually, the patient died of aggressive disease 16 days after haploidentical hematopoietic stem-cell transplantation (HSCT) with her father as the donor. One male patient with stage II BL had a local relapse on the nasopharynx after the completion of the initial chemotherapy with the POG-9219 protocol. He restarted treatment with the POG-9317 protocol and additional radiation therapy (30 Gy delivered in 15 fractions to the local site); however, he died of respiratory failure due to progressive lymphoma. A 4-year-old female patient with stage III DLBCL started treatment with the R-CHOP protocol. After 9 cycles of the R-CHOP protocol, she developed fever, abdominal pain, and pancytopenia and was evaluated for relapse. BM biopsy showed evidence of BM involvement of the DLBCL and findings of hemophagocytosis. The patient was newly diagnosed with hemophagocytic lymphohistiocytosis (HLH) because she satisfied $>5$ of the 8 HLH criteria. She completed therapy with 10 cycles of the R-CHOP protocol followed by the HLH2004 protocol; however, she eventually died of acute respiratory distress syndrome associated with cytomegalovirus infection during the HLH therapy. 
2) Death

Three patients died without experiencing disease relapse. One patient with stage IV BL died of gastrointestinal tract bleeding due to chemotherapy-induced thrombocytopenia (treatment-related death), and one patient with stage IV DLBCL died of intracranial hemorrhage due to refractory thrombocytopenia associated with uncontrolled disease. Another patient with stage III BL died of intestinal obstruction during induction.

3) Secondary malignancy

During the follow-up period, 2 patients were diagnosed with secondary malignancies after the initial treatment. A 12-year-old male patient had an ileocecal mass and underwent surgical resection. He was diagnosed with stage IV BL without CNS involvement. He received the LMB96 group C regimen as chemotherapy and achieved complete remission (CR) without acute complications. At 36 months from the initial diagnosis, BM examination was performed because of fever, recurrent oral ulcers, and thrombocytopenia. The patient was diagnosed with therapy-related acute myeloid leukemia and treated with the Medical Research Council Acute Myeloid Leukemia 12 protocol [18], followed by matchedsibling HSCT. He was still alive 1 year after HSCT, with complications of acute graft-versus-host disease. He was treated with prednisolone for 1 month, followed by cyclosporine as secondary treatment for 1 year.

Another 12-year-old female patient with DLBCL had multiple masses on the lymph nodes, bones, kidneys, and pancreas at diagnosis. She underwent initial chemotherapy with the LMB96 group B regimen; however, multiple lesions suspicious for residual bone involvement were detected on bone scan and PET-CT after the second cycle of maintenance chemotherapy. For salvage treatment, total body irradiation with cyclophosphamide and etoposide conditioning and autologous HSCT were performed. An endocrinologic evaluation was conducted 9 years after HSCT because of low body mass index and amenorrhea. On thyroid ultrasonography, a mass measuring approximately $3 \mathrm{~cm}$ was detected in the left thyroid gland and follicular thyroid carcinoma was finally diagnosed after left hemithyroidectomy. She was still alive 12 years after the initial diagnosis. 


\section{Rituximab with LMB chemotherapy}

Two patients were treated with the LMB96 protocol added with rituximab as the initial treatment. A 4-year-old male patient with stage IV BL classified as group C had right ophthalmoplegia and facial palsy at diagnosis. He received chemotherapy according to the protocol of Goldman et al. [19]. Rituximab $\left(375 \mathrm{mg} / \mathrm{m}^{2} /\right.$ dose) was administered on days -2 and 0 of each of the two induction cycles and on day 0 of each the two consolidation cycles (6 total doses). He achieved CR without chemotherapy-related adverse events and was still alive 19 months after the initial diagnosis.

A 6-year-old female patient with stage IV group C BL had multiple masses in the bone and lymph nodes with BM involvement at diagnosis. She underwent chemotherapy with the LMB96 protocol and received 2 doses of rituximab during the consolidation phase. She also achieved CR and was still alive without complications after 29 months.

\section{DISCUSSION}

Mature B-cell lymphoma is a common type of NHL. It presents as a high-grade and aggressive tumor in the pediatric population. Several research groups have conducted various studies on chemotherapy for NHL, and the treatment strategies are complex. The prognosis of pediatric mature B-cell lymphoma has substantially improved over the decades, with good long-term survival. In 2020, the National Comprehensive Cancer Network (NCCN) guidelines for pediatric aggressive mature Bcell lymphomas provided guidance on the pathology and diagnosis, staging, initial treatment, and therapy for relapsed or refractory disease [1]. Our retrospective study reviewed the clinical characteristics of patients, outcomes of various protocols, and treatments of relapsed patients with mature B-cell lymphoma over 28 years at a single institute.

The clinical trials by the LMB, BFM, and CCG/COG groups reported EFS rates of $>90 \%$ in earlystage disease and $67-79 \%$ in advanced-stage disease $[3,6,12,20]$. In our study, the overall OS and EFS rates in our patients were $92.7 \%$ and $86.6 \%$, respectively. Even when compared according to stage, the OS and EFS rates were $96.2 \%$ and $93.3 \%$ for stage I-II and $90.4 \%$ and $82.7 \%$ for stage IIIIV, respectively. These results were comparable to those of previous studies. However, the treatment 
outcomes are still poor for advanced BL or DLBCL, and no standard treatment has been suggested for salvage therapy, especially in cases of relapse [21]. In our study, stage IV DLBCL particularly had a very poor outcome even with the LMB group C protocol. The 5-year OS of patients in group C was $85.7 \%$, with a statistically significant difference between patients in group $\mathrm{C}$ and those in groups $\mathrm{A}$ and B.

For these high-risk patients, rituximab combination therapy has been reported to provide a good outcome. Rituximab is a chimeric murine/human monoclonal antibody with a high affinity against CD20 in B cells. In adults with B-NHL, rituximab has shown efficacy and has become a standard component of care [22]. Goldman et al. [19] suggested the safety and improved efficacy of therapy with the addition of rituximab compared with the standard LMB96 protocol in pediatric patients with BL who had BM ( $\geq 25 \%$ blasts) or CNS involvement. The efficacy of rituximab in high-risk patients classified as group B was also supported by Goldman et al. [23]. In this trial, patients with stage III/IV and group B mature B-NHL who received rituximab with the FAB/LMB96 protocol showed an improvement in the 3-year EFS rate (95\% in the R-LMB group), without serious adverse events.

Recently, Minard-Colin et al. [8] conducted a randomized trial in 328 patients aged $<18$ years with high-risk (stage III with an elevated LDH level or stage IV) mature B-NHL to compare the R-LMB protocol with the standard LMB protocol alone. They reported markedly improved 3-year EFS and OS rates of $>93 \%$ in the R-LMB group. On the basis of these reports, the NCCN guideline recommended rituximab-combined chemotherapy in high-risk patients classified as groups B and C [1]. In our study, 2 patients underwent therapy with the combination of rituximab and the LMB96 protocol, both of whom showed good treatment outcomes without treatment-related complications.

In HGBL, a new separate entity with $M Y C, B C L 2$, or BCL6 rearrangements, also known as doublehit or triple-hit lymphoma, has been defined in the 2016 revised WHO classification of lymphoid neoplasms [2]. Currently, HGBL with MYC and BCL2 translocations accounts for approximately $10 \%$ of all lymphomas and occurs in older patients (median age, $60 \mathrm{yr}$; range, 6-82 yr) [24]. Although several studies reported such cases as a poor prognostic group in both pediatric and adult patients [25, 26], the recommended treatment for double-hit and triple-hit lymphomas in the pediatric population is 
similar to that for other high-grade mature B-cell lymphomas. Among 51 patients with available immunohistochemistry or fluorescence in situ hybridization data in our study, 6 patients were suspicious for double-hit lymphoma with $M Y C$ and BCL6 rearrangements. The survival outcomes in this group could not be analyzed owing to the small number of patients; however, only 1 death occurred, in a 4-year-old patient with stage IV BL treated with the LMB96 regimen who had relapse in the CNS and BM and eventually died of progressive lymphoma. Future extensive studies on the prognosis of double-hit and triple-hit lymphomas in pediatric patients with high-grade mature B-cell lymphoma are needed.

The long-term treatment outcomes in pediatric patients with mature B-cell lymphoma have considerably improved over the recent decades. Particularly, a gradual increase in the EFS rate was achieved by eliminating local and CNS radiotherapy, reducing the total doses of cyclophosphamide and doxorubicin, and, recently, adding rituximab [27]. Nevertheless, treatment-related toxicity and mortality remain important concerns and less toxic treatment modalities are continuously being studied. Cairo et al. reported a toxicity-related death rate of 3\%, with a high incidence of grade III/IV mucositis, infection, myelosuppression, and prolonged hospitalization during the induction courses [12]. Tumor lysis syndrome (TLS) is also a well-known treatment-related toxicity, especially in BL, due to the high turnover rate of tumor cells. To prevent TLS, vigorous alkaline diuresis needs to be obtained, with furosemide, if necessary, and urate oxidase needs to be administered before the initiation of treatment. BL/B-cell acute lymphoblastic leukemia and LDH levels $\geq 500 \mathrm{U} / \mathrm{L}$ were associated with the highest incidence of TLS among patients with NHL (14.9\%) [28]. In patients treated with the LMB89 protocol, the use of urate oxidase reduced mortality due to TLS. In our study, of the total of 50 patients with BL, 15 had TLS (30.0\%) and all of them had stage III/IV disease. Among the 11 patients with TLS who underwent continuous renal replacement therapy, no cases of TLS-related death occurred. Conversely, treatment-related mortality occurred in 1 male patient during consolidation chemotherapy. He died of gastrointestinal bleeding caused by myelosuppression-related thrombocytopenia.

Secondary malignancy after the treatment of pediatric NHL is also a concern for long-term 
treatment outcomes. In the North American Childhood Cancer Survivor Study cohort, 229 secondary malignant neoplasms (SMNs) occurred among 206 survivors treated with chemotherapy alone after a childhood cancer diagnosis [29]. The survivors had a 1.9-fold, 4.6-fold, and 3.8-fold increased standardized incidence ratio of subsequent leukemia/lymphoma, breast cancer, and thyroid cancer, respectively. Recently, Ehrhardt et al. reported the occurrence of SMNs in children and adolescents with NHL by investigating a total of 570 survivors over 5 years after the initial diagnosis. None of the 126 survivors treated with the contemporary LMB protocol between 1987 and 1999 developed SMNs. However, patients treated with non-LMB protocols had an SMN incidence rate of 2.2 per 1,000 person-years $(5,871$ total person-years) [30]. Two cases of SMN were observed in our study: therapyrelated acute myeloid leukemia and thyroid cancer. These were successfully treated with allogeneic HSCT and surgery.

Our study had several limitations. First, it was a retrospective study with a small sample of patients recruited from a single center. Second, as the criteria for selecting the treatment strategy have changed over the last 25 years, various treatment regimens were applied with different indications. Therefore, the number of patients significantly differed among the different treatment protocols, making it difficult to compare the treatment outcomes. In addition, detailed data on treatment-related toxicities for each chemotherapy regimen were not available.

In summary, this study revealed favorable treatment outcomes in children and adolescents with aggressive mature B-cell lymphoma despite the long study period ( $>25 \mathrm{yr}$ ). However, further studies are needed to improve the survival rate of patients with advanced-stage disease or disease relapse. Targeted therapies such as rituximab are expected to become more widely used to improve the outcomes of high-risk patients. In addition to efforts to improve the survival rates, careful monitoring for acute toxicity or secondary malignancy due to intensive multidrug chemotherapy is required. The development of guidelines for the follow-up observation of these patients may help in monitoring chemotherapy-related complications. 
Authors' Disclosure of Potential Conflict of Interest

No potential conflicts of interest relevant to this article were reported. 


\section{REFERENCES}

1. Davies K, Barth M, Armenian S, et al. Pediatric aggressive mature B-cell lymphomas, version 2.2020, NCCN Clinical Practice Guidelines in Oncology. J Natl Compr Canc Netw 2020;18:1105-23.

2. Swerdlow SH, Campo E, Pileri SA, et al. The 2016 revision of the World Health Organization classification of lymphoid neoplasms. Blood 2016;127:2375-90.

3. Gerrard M, Cairo MS, Weston C, et al. Excellent survival following two courses of COPAD chemotherapy in children and adolescents with resected localized B-cell non-Hodgkin's lymphoma: results of the FAB/LMB 96 international study. Br J Haematol 2008;141:840-7.

4. Cairo MS, Beishuizen A. Childhood, adolescent and young adult non-Hodgkin lymphoma: current perspectives. Br J Haematol 2019;185:1021-42.

5. Patte C, Auperin A, Michon J, et al. The Société Française d'Oncologie Pédiatrique LMB89 protocol: highly effective multiagent chemotherapy tailored to the tumor burden and initial response in 561 unselected children with B-cell lymphomas and L3 leukemia. Blood 2001;97:3370-9.

6. Patte C, Auperin A, Gerrard M, et al. Results of the randomized international FAB/LMB96 trial for intermediate risk B-cell non-Hodgkin lymphoma in children and adolescents: it is possible to reduce treatment for the early responding patients. Blood 2007;109:2773-80.

7. El-Mallawany NK, Cairo MS. Advances in the diagnosis and treatment of childhood and adolescent B-cell non-Hodgkin lymphoma. Clin Adv Hematol Oncol 2015;13:113-23.

8. Minard-Colin V, Aupérin A, Pillon M, et al. Rituximab for high-risk, mature B-cell nonHodgkin's lymphoma in children. N Engl J Med 2020;382:2207-19.

9. Moleti ML, Testi AM, Foà R. Treatment of relapsed/refractory paediatric aggressive B-cell non-Hodgkin lymphoma. Br J Haematol 2020;189:826-43. 
10. Simonin M, Auperin A, Bertrand Y, et al. In childhood mature B-NHL with CNS disease, patients with blasts in cerebrospinal fluid are at higher risk of failure. Blood Adv 2020;4:3621-5.

11. Murphy SB. Classification, staging and end results of treatment of childhood non-Hodgkin's lymphomas: dissimilarities from lymphomas in adults. Semin Oncol 1980;7:332-9.

12. Cairo MS, Gerrard M, Sposto R, et al. Results of a randomized international study of high-risk central nervous system B non-Hodgkin lymphoma and B acute lymphoblastic leukemia in children and adolescents. Blood 2007;109:2736-43.

13. Anderson JR, Jenkin $\mathrm{RD}$, Wilson JF, et al. Long-term follow-up of patients treated with COMP or LSA2L2 therapy for childhood non-Hodgkin's lymphoma: a report of CCG-551 from the Childrens Cancer Group. J Clin Oncol 1993;11:1024-32.

14. Link MP, Shuster JJ, Donaldson SS, Berard CW, Murphy SB. Treatment of children and young adults with early-stage non-Hodgkin's lymphoma. N Engl J Med 1997;337:1259-66.

15. Hutchison RE, Laver JH, Chang M, et al. Non-anaplastic peripheral T-cell lymphoma in childhood and adolescence: a Children's Oncology Group study. Pediatr Blood Cancer 2008;51:29-33.

16. Laver JH, Kraveka JM, Hutchison RE, et al. Advanced-stage large-cell lymphoma in children and adolescents: results of a randomized trial incorporating intermediate-dose methotrexate and high-dose cytarabine in the maintenance phase of the APO regimen: a Pediatric Oncology Group phase III trial. J Clin Oncol 2005;23:541-7.

17. Pui CH, Campana D, Pei D, et al. Treating childhood acute lymphoblastic leukemia without cranial irradiation. N Engl J Med 2009;360:2730-41.

18. Burnett AK, Hills RK, Milligan DW, et al. Attempts to optimize induction and consolidation treatment in acute myeloid leukemia: results of the MRC AML12 trial. J Clin Oncol 2010;28:586-95. 
19. Goldman S, Smith L, Galardy P, et al. Rituximab with chemotherapy in children and adolescents with central nervous system and/or bone marrow-positive Burkitt lymphoma/leukaemia: a Children's Oncology Group report. Br J Haematol 2014;167:394-401.

20. Song JS, Youn HS, Im HJ, Ghim T, Moon HN, Seo JJ. Treatment outcome and prognostic factors for children with advanced non-Hodgkin's lymphoma at a single institution. Korean J Hematol 2006;41:157-66.

21. Harker-Murray PD, Pommert L, Barth MJ. Novel therapies potentially available for pediatric B-cell non-Hodgkin lymphoma. J Natl Compr Canc Netw 2020;18:1125-34.

22. Lee HG, Choi Y, Kim SY, et al. R-CHOP chemoimmunotherapy followed by autologous transplantation for the treatment of diffuse large B-cell lymphoma. Blood Res 2014;49:107-14.

23. Goldman S, Smith L, Anderson JR, et al. Rituximab and FAB/LMB 96 chemotherapy in children with stage III/IV B-cell non-Hodgkin lymphoma: a Children's Oncology Group report. Leukemia 2013;27:1174-7.

24. Li S, Seegmiller AC, Lin P, et al. B-cell lymphomas with concurrent MYC and BCL2 abnormalities other than translocations behave similarly to MYC/BCL2 double-hit lymphomas. Mod Pathol 2015;28:208-17.

25. Uemura S, Hasegawa $\mathrm{D}$, Yokoi $\mathrm{T}$, et al. Refractory double-hit lymphoma/leukemia in childhood mimicking B-precursor acute lymphoblastic leukemia at initial presentation. Rinsho Ketsueki 2017;58:143-9.

26. Thirunavukkarasu B, Samanta J, Bhatia P, Bal A. De novo double-hit B-cell precursor leukemia/lymphoma - an unusual presentation as peritoneal lymphomatosis. Autops Case Rep 2021;11:e2021278.

27. Frazer JK, Li KJ, Galardy PJ, et al. Excellent outcomes in children and adolescents with CNS + Burkitt lymphoma or other mature B-NHL using only intrathecal and systemic chemoimmunotherapy: results from FAB/LMB96 and COG ANHL01P1. Br J Haematol 2019;185:374-7. 
28. Wössmann W, Schrappe M, Meyer U, Zimmermann M, Reiter A. Incidence of tumor lysis syndrome in children with advanced stage Burkitt's lymphoma/leukemia before and after introduction of prophylactic use of urate oxidase. Ann Hematol 2003;82:160-5.

29. Turcotte LM, Liu Q, Yasui Y, et al. Chemotherapy and risk of subsequent malignant neoplasms in the Childhood Cancer Survivor Study cohort. J Clin Oncol 2019;37:3310-9.

30. Ehrhardt MJ, Chen Y, Sandlund JT, et al. Late health outcomes after contemporary Lymphome Malin de Burkitt therapy for mature B-cell non-Hodgkin lymphoma: a report from the Childhood Cancer Survivor Study. J Clin Oncol 2019;37:2556-70. 
Table 1. Characteristics of the patients.

\begin{tabular}{ll}
\hline Characteristics & $\mathrm{N}=82(\%)$
\end{tabular}

Age at diagnosis, median (range, yr)

$7.8(1.3-16.4)$

Sex

Male

Female

\section{Primary site}

Head and neck, except skin and nodes

Thorax

$5(6.1)$

Abdomen

$43(52.4)$

Peripheral lymph nodes

$11(13.4)$

Central nervous system

$2(2.4)$

Others

\section{Pathologic subtype}

Burkitt lymphoma

$50(61.0)$

Diffuse large B-cell lymphoma

29 (35.4)

High-grade B-cell lymphoma, not otherwise specified

$3(3.6)$

\section{BM involvement}

Yes

No

\section{CNS involvement}

Yes

No

\section{Stage}

I

II

III

$30(36.6)$ 
Chemotherapy protocol and group

LMB89/LMB96

$68(82.9)$

Group A

$6(8.8)$

Group B

$35(51.5)$

Group C

$27(39.7)$

POG-9219/9315/9317

$9(11.0)$

D-COMP/COMP

$3(3.7)$

R-CHOP/CHOP

$2(2.4)$

Abbreviations: BM, bone marrow; CNS, central nervous system. 
Table 2. Characteristics of the events.

\begin{tabular}{lr}
\hline Event characteristics & \\
\hline Total events ( $\mathrm{N}$ of patients) & 11
\end{tabular}

$\begin{array}{lr}\text { Relapse (N) } & 6 \\ \text { Local relapse } & 4 \\ \text { BM relapse } & 1 \\ \text { CNS relapse } & 1 \\ \text { Median time to relapse (range, mo) } & 6.8(4.3-13.9) \\ \text { Pathologic subtype } & 2(33.3 \%) \\ \text { Burkitt lymphoma } & 4(66.7 \%) \\ \text { Diffuse large B-cell lymphoma } & \\ \text { Stage } & 2(33.3 \%) \\ \text { II } & 3(50.0 \%) \\ \text { III } & 1(16.7 \%) \\ \text { IV } & \\ \end{array}$

Salvage regimen
CTx only
$4(66.7 \%)$
CTx+RTx
$1(16.7 \%)$
CTx+RTx+allo-HSCT
$1(16.7 \%)$

$\begin{array}{ll}\text { Death after relapse (N) } & 4\end{array}$

Death of any cause other than relapse (N) 3

$\begin{array}{ll}\text { Progressive disease } & 2\end{array}$

Treatment related 1

Median time to death (range, mo) $10.5(3.7-27.1)$

$\begin{array}{ll}\text { Secondary malignancy }(\mathrm{N}) & 2\end{array}$

Therapy-related acute myeloid leukemia 1

Follicular thyroid carcinoma $\quad 1$ 
Abbreviations: BM, bone marrow; CNS, central nervous system; CTx, chemotherapy; HSCT, hematopoietic stem cell transplantation; RTx, radiotherapy. 


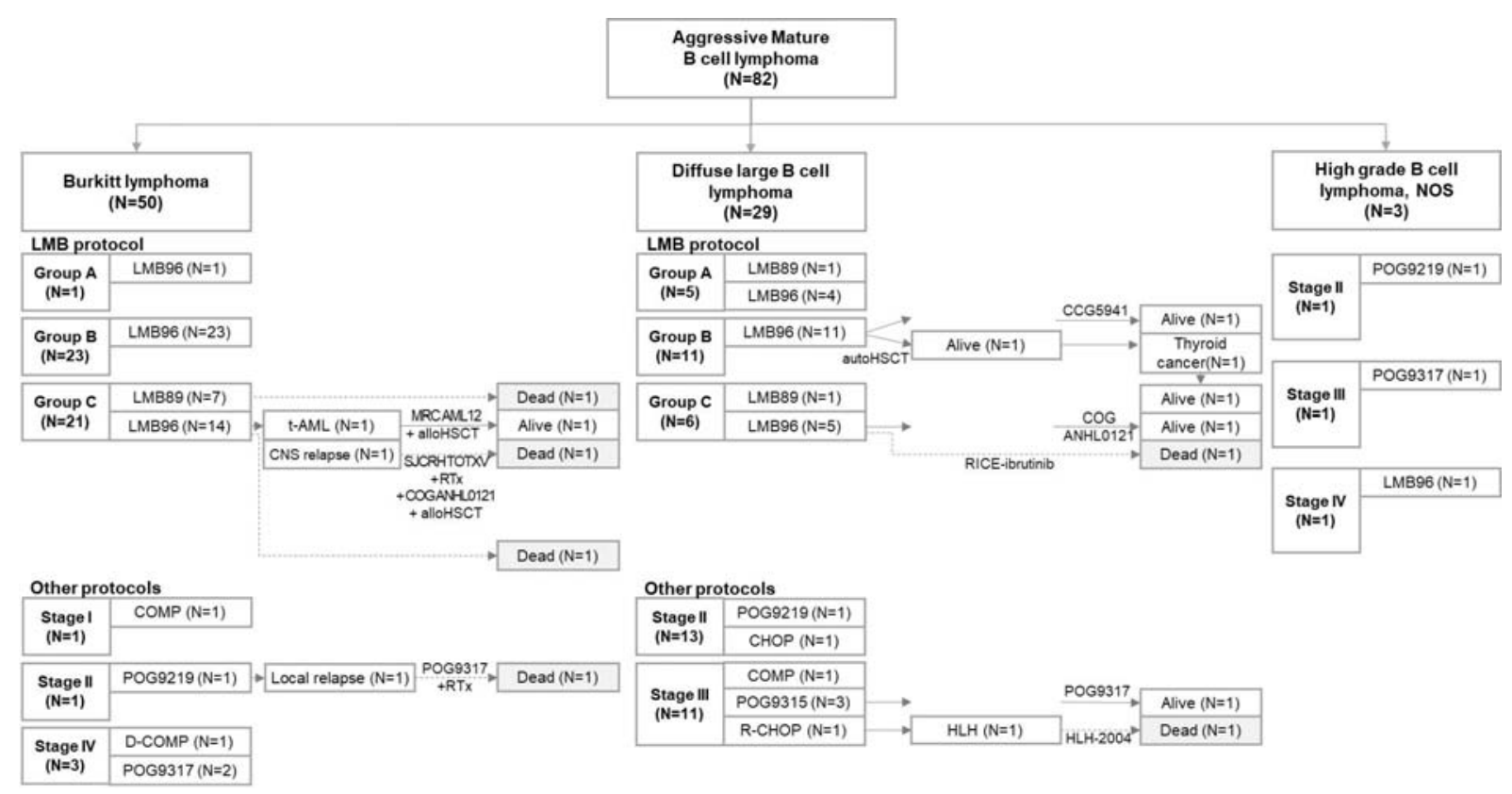

Fig. 1. Events and outcomes according to treatment protocol.

Abbreviations: HLH, hemophagocytic lymphohistiocytosis; HSCT, hematopoietic stem cell transplantation; NOS, not otherwise specified; RTx, radiotherapy; t-AML, therapy-related acute myeloid leukemia. 


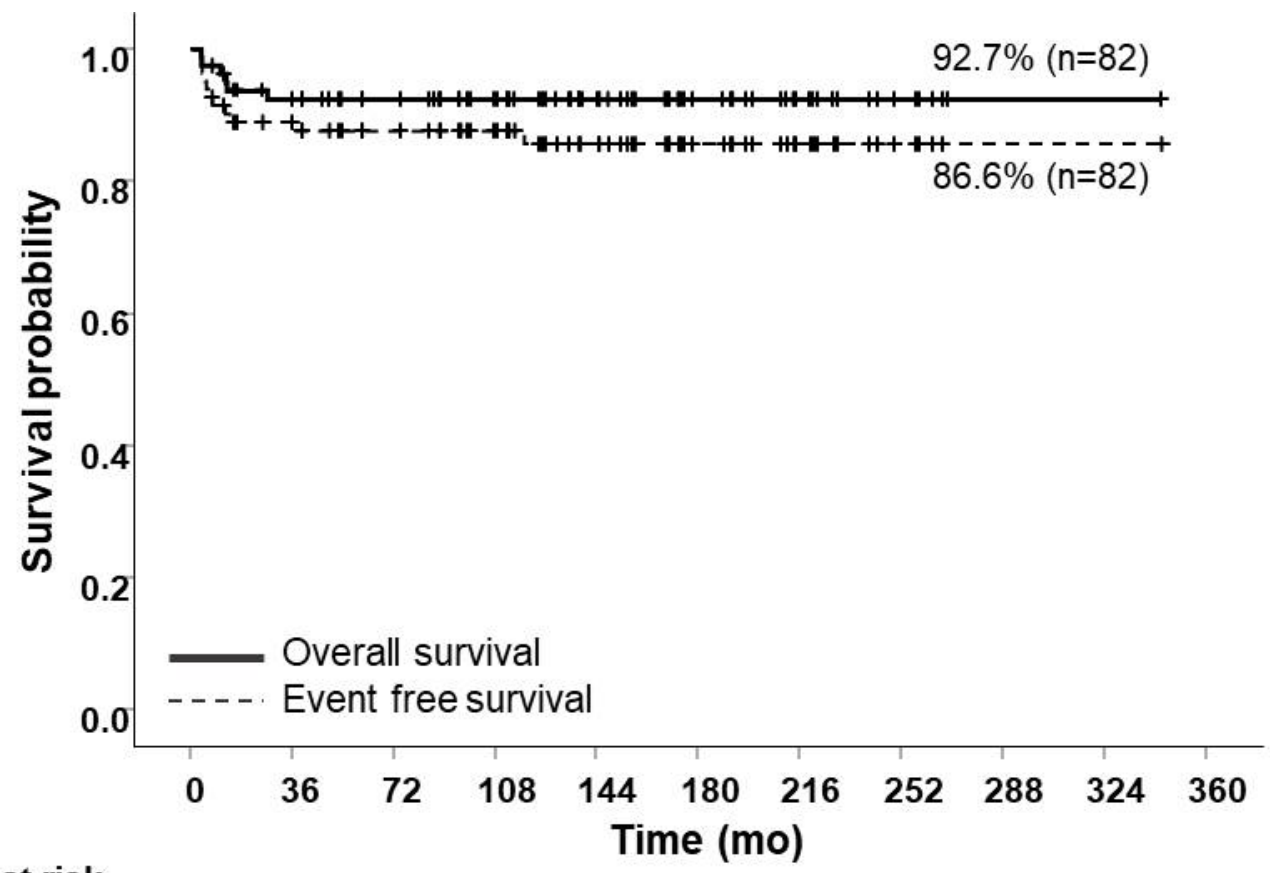

No. at risk

$\begin{array}{llllllllllll} & 82 & 71 & 63 & 53 & 38 & 24 & 15 & 7 & 2 & 2 & 0 \\ -\ldots- & 82 & 68 & 60 & 51 & 37 & 23 & 14 & 6 & 2 & 2 & 0\end{array}$

Fig. 2. Survival outcomes of the 82 patients. 

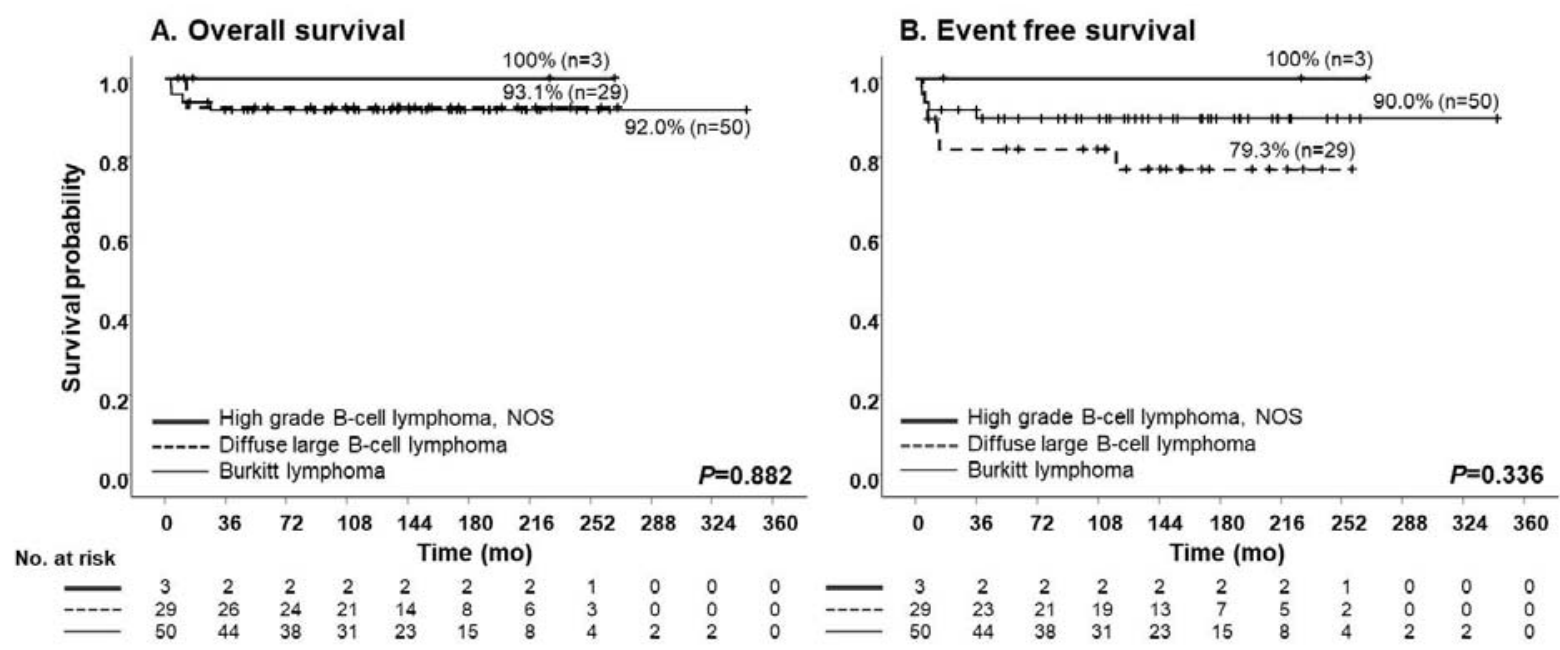

Fig. 3. Treatment outcomes according to histopathologic subtype: overall survival (A) and event-free survival (B) rates.

Abbreviation: NOS, not otherwise specified. 


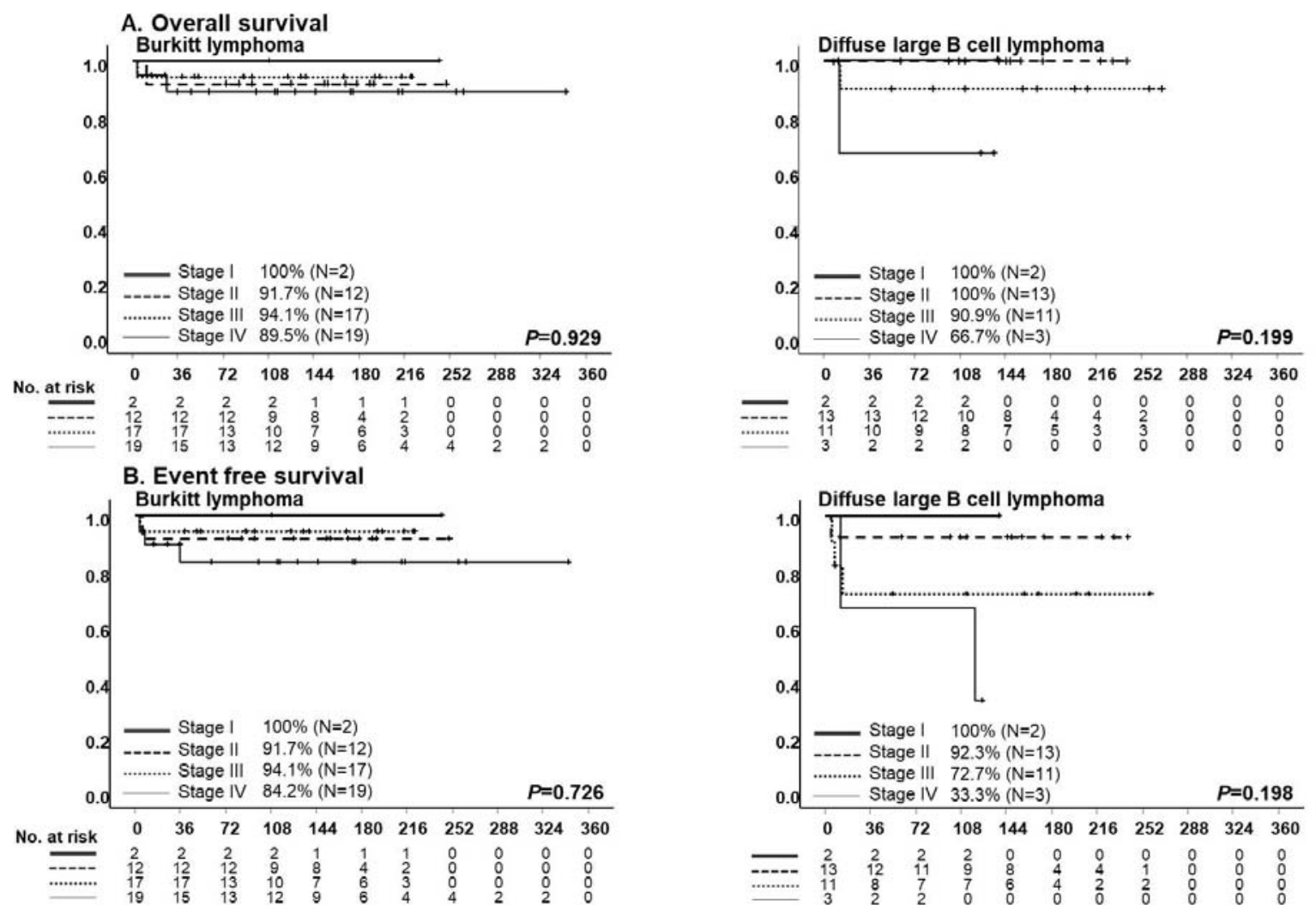

Fig. 4. Overall survival (A) and event-free survival (B) rates of patients with Burkitt lymphoma and diffuse large B-cell lymphoma according to initial stage. 
A. Overall survival

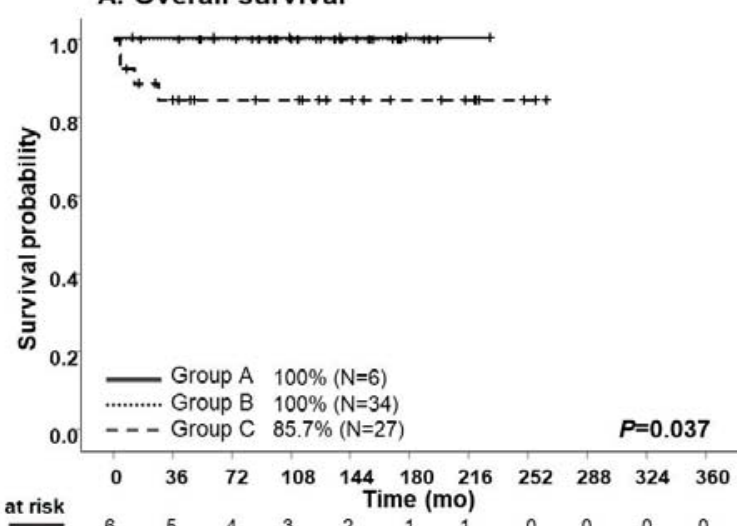

No. at risk
B. Event free survival

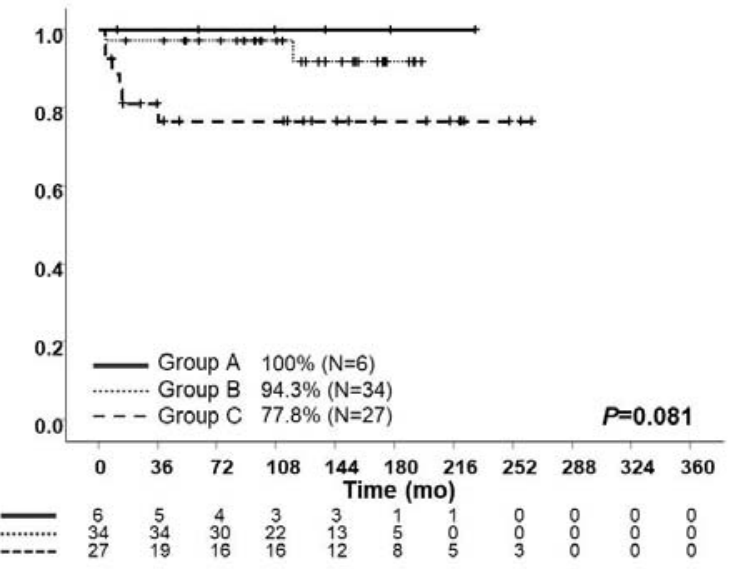

Fig. 5. Survival outcomes of patients with Burkitt lymphoma and diffuse large B-cell lymphoma treated with the LMB protocols according to risk group classification: overall survival (A) and event-free survival (B) rates. 

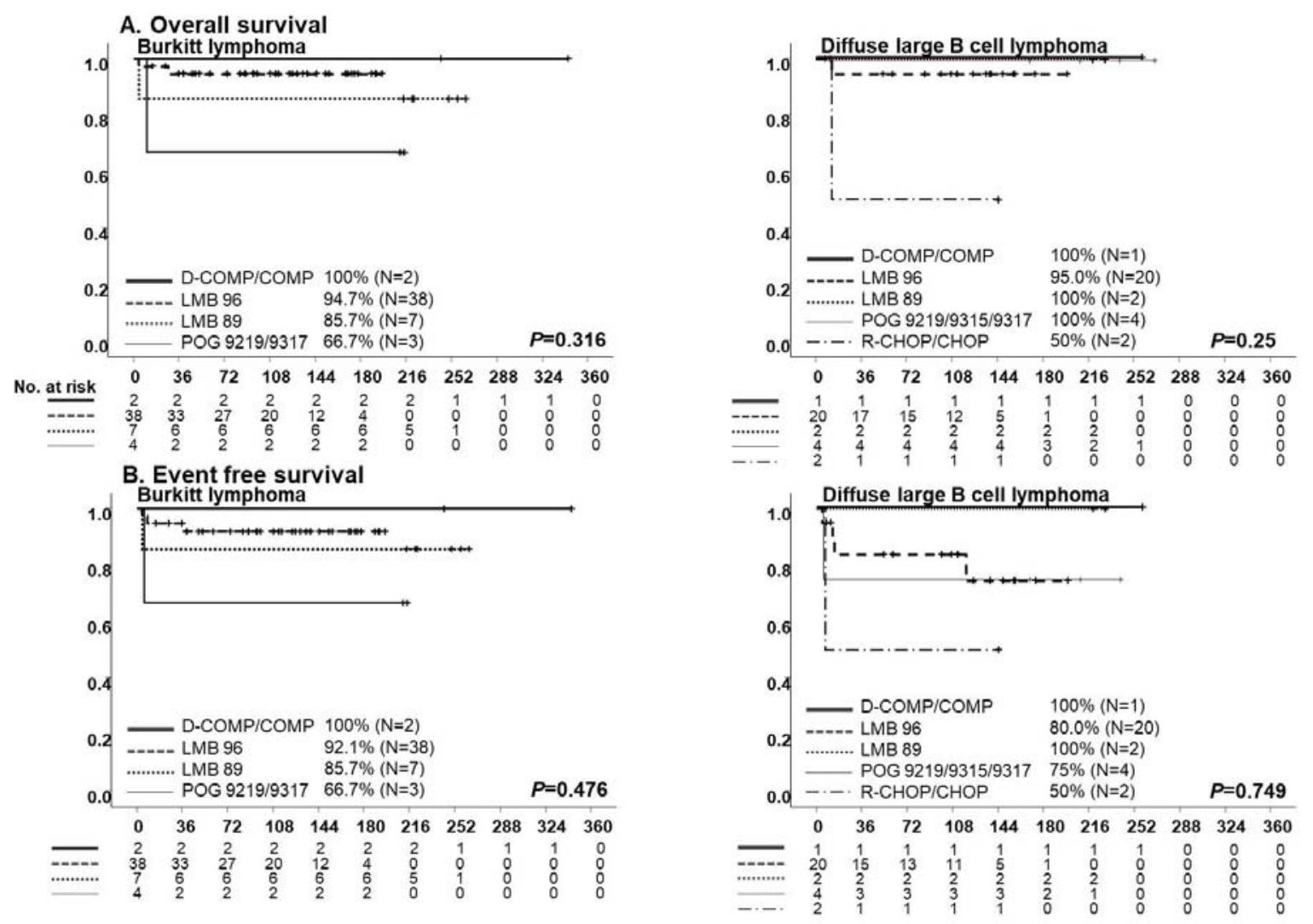

Fig. 6. Survival outcomes of patients with Burkitt lymphoma and diffuse large B-cell lymphoma according to the initial chemotherapy: overall survival (A) and event-free survival (B) rates. 